\title{
DEVICE FOR MEASURING BDF - SPATIAL - Spectral Light - Scattering Properties of SURFACES
}

\author{
Letunov, A.; BARladian, B.; GAlaKtionov,V.; Ershov, S.; \\ Voloboy, A. \& ZuEVA, E.
}

Abstract: Device for measuring spectral - spatial distribution of light dispersed by surfaces is presented. These data are necessary for calculations and computer simulation of reflectors, liquid crystal displays, clothes, paints, and other objects and materials with non trivial light-scattering characteristics. The modern systems of computer graphics also need such data for accurate simulation of light propagation. The direct measurements are the more reliable methods to obtain these data, but this task is not easy. Conception proposed by authors allowed to realize the effective device, which is used for numerous practical tasks.

Key words: computer graphics, light scattering, non-uniform grid, high-lighted zone
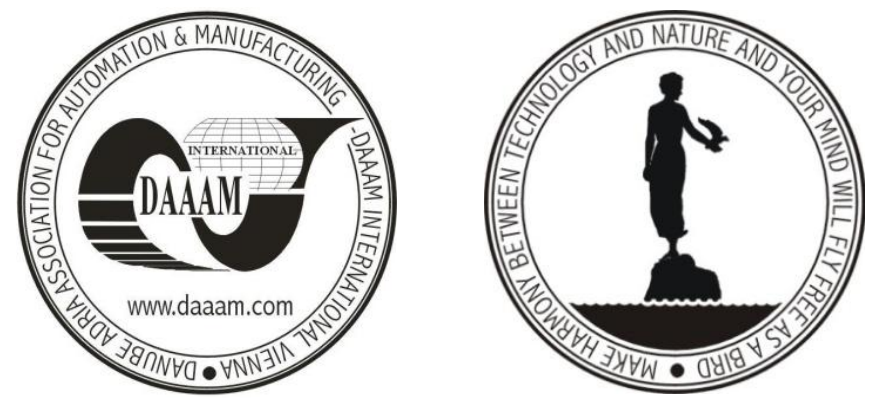

Authors' data: Dr. Letunov, A[lexandr]*; Dr. Barladian, B[oris]**; Prof. Galaktionov, V[ladimir]**; Dr. Ershov, S[ergey]**; Dr. Voloboy, A[lexey]**; Dr. Zueva, E[lena]**, *Prokhorov General Physics Institute, Russian Academy of Sciences, 119991, Moscow, Vavilov Str., 38,** Keldysh Institute of Applied Mathematics, Miusskaya sq., 4 Moscow, 125047, let@fpl.gpi.ru, obb@gin.keldysh.ru, $\quad$ vlgal@gin.keldysh.ru, $\quad$ ersov@integra.jp, voloboy@gin.keldysh.ru, ezueva@keldysh.ru

This Publication has to be referred as: Letunov, A[lexandr]; Barladian, $\mathrm{B}$ [oris]; Galaktionov, V[ladimir]; Ershov, S[ergey]; Voloboy, A[lexey] \& Zueva, E[lena] (2012). Device for Measuring BDF - Spatial - Spectral Light - Scattering Properties of Surfaces, Chapter 40 in DAAAM International Scientific Book 2012, pp. 479-490, B. Katalinic (Ed.), Published by DAAAM International, ISBN 978-3-901509-86-5, ISSN 1726-9687, Vienna, Austria

DOI: 10.2507/daaam.scibook.2012.40 


\section{Introduction}

A device for measuring the spectral and spatial distribution of light reflected from flat surfaces is described in the present work. These data characterize the properties of materials and are used to model objects containing light-scattering surfaces. For example, they are necessary in lighting engineering for modeling complicated optical devices such as reflectors and elements of liquid-crystal panels, and in such applications of today as modeling of clothes, paint-coated surfaces, decoration materials, etc. Modern means of generating photorealistic images are also based on the knowledge of the light-scattering properties of the materials as well as on physically accurate simulation of light propagation. Direct measurements are the most reliable way to study the light-scattering properties of materials. The task is not easy due to the large amount of data and to the existence of special directions in which the gradient of the measured characteristics is high. One of the main requirements to the measuring device is that it is capable to record data from many directions, and at the same time to provide a high spatial resolution in the critical region near specular reflection. To this effect, the angular distribution of light recorded on directions close to specular reflection is converted into spatial distribution and is recorded as an image. Optical fibers are used to gather the light reflected in other directions. A CCD-camera serves as the light receiver.

The most common way to describe the light-scattering properties of a surface is to define its Bidirectional Reflectance / Transmittance Distribution Function (BRDF/BTDF) [Horn, 1985]. Defined in a number of points on the surface, the $\mathrm{BRDF} / \mathrm{BTDF}$ indicates which part of the energy received by the surface from a direction $K_{\mathrm{i}}$ will be reflected (and accordingly, transmitted) by the surface in another direction $K_{\mathrm{s}}$. This dependence can be graphically shown as a number of indicatrices for reflection/transmission for several incident angles. In general case, BRDF/BTDF is a function of five variables, four spatial ones specifying two directions, and a spectral one. For isotropic surfaces, there are only three spatial variables, one defines the direction of incident beam, and two others the direction of viewing. For brevity, from now on we will be calling this function BDF in both cases where it does not cause misunderstanding. Normally, BDF is presented in table form with uniform or non-uniform grid of input data.

It is rather difficult to measure the BDF. Different approaches to this task are described in [Ward, 1992], [Karner et al., 1996], [Foo et al., 1997]. A number of research centers have created laboratories that carry out such measurements [Matusik et al., 2003], [Yasuhiro Mukaigava et al., 2007].

In the present work, we describe our concept for measuring BDF, the device that was created for this purpose, and the conclusions that were drawn after the device had been operating for a period of time. This work is a continuation of studies [Letunov et al., 1999], [Letunov et al.,2000], [Voloboy, Vyukova et al., 2006], [Letunov et al., 2011]. Since then, the accuracy and operation speed of the device were increased, its dynamic range was enlarged, and some of the other characteristics also were optimized. To this end, we started using a better image detector: a monochrome digital video camera with 14-bit AD converter, and a better spectral 
device. It is the SP2150 monochromator with dual grating turret equipped with two interchangeable gratings, the dispersion of one of which was twice that of the other. The sensitivity of the monochromator increases when using the grating with lesser dispersion, yet the spectral resolution decreases.

In order to fully realize the new capacities of the device, we needed to modify its design. In turn, this modification gave the possibilities to apply a more effective algorithm for processing the video information. The description of the computational scheme of data processing and its realization is given in [Voloboy, V'ukova et al., 2005], [Voloboy et al., 2006].

\section{Device description.}

Our object when creating the device was to minimize the measurement time while achieving results accuracy and reliability that would be sufficient for practical needs. The most of studied surfaces have a high gradient of the BRDF for the observation angles near the direction of specular reflection and of the BTDF for direct transmission. Therefore, the construction of the device and the measurement method should allow for high angular resolution in the mentioned regions and a good coverage of the solid angle into which light is reflected.

To meet the requirement that the density of points is high in selected directions at the same time that the measurement time is low, we use an image of the reflected light obtained with a digital camera.

In the device described in pioneering work [Ward, 1992], a digital camera is also used to show all the directions of scattering, the space being "turned out" using a reflecting sphere. The camera is placed near the sample and uses a wide-angle "fisheye" lens. This solution, however, leads to a high level of parasitic scattering of light on the elements of the device, which negatively affects the reliability and accuracy of the results.

In the present work, we decided to place the camera separately from the sample. A lens is used to gather the light reflected in the maximum resolution region, and optical fibers in other directions. The sample is illuminated by a parallel beam of light with a narrow wavelength range. The light reflected by the sample is measured simultaneously in many ( 400) directions.

Figure 1 presents the schematic of the device.

The device consists of a system that forms the incident beam, a movable base to which is affixed the sample, and an optical system to gather and record the reflected light. The light-gathering system includes a blackened semi-sphere, a screen with a plate from matted glass and the output ends of the optical fibers, and a CCD camera which is used to record the light. The semi-sphere, whose internal radius is $200 \mathrm{~mm}$, with holes made in it for the incident beam, serves as the rest for the lens and the output ends of the optical fibers. Let us consider the construction of the device following the path of the light beam.

A xenon high pressure ozone-free arc lamp DKsShIL-150 serves as the source of light. The lamp power is 150 watt; effective arc size is $1.0 \times 0.3 \mathrm{~mm}$, and color temperature is about $6000 \mathrm{~K}$. The image of the central region of the arc is magnified 
two times by the input collimator (lens1) made in the form of a lens with iris diaphragm. This image is then projected onto the input slit of the computercontrolled Princeton Instruments SP2150 monochromator with aperture ratio f/4.0. The dispersion of the monochromator depends on the chosen grating and is 10 $\mathrm{nm} / \mathrm{mm}$ for one and $20 \mathrm{~nm} / \mathrm{mm}$ for the other. The diaphragm allows regulating the angular dimensions of the incident beam. During the measurements, the monochromator scans the visible spectrum by dividing it into several tens of $10-\mathrm{nm}$ wide spectral fragments in the range $390-730 \mathrm{~nm}$. A different pace can be used when necessary, both divisible by $10 \mathrm{~nm}$ and other as allowed by the construction of the monochromator. The software that controls the device allows choosing different measurement ranges and different widths of the spectral channel. It is possible to choose an irregular coverage of the range and its broadening depending on the object of the measurements. The limits are defined by the sensitivity of the system, and the knowledge of the light-scattering properties of the sample and the transmission of the filters. In the current release, the limit on the blue (short-wave) side was defined by the drop of sensitivity of the camera with the increase of the absorption in the optical fiber with the decrease of wave length. On the red (long-wave) side, the limit was defined by the passport data. The allowed spectral range covers the entirety of the spectral region visible to the human eye. Normally, during the measurement process the monochromator scans the visible area of the spectrum, subdividing it into 3510 nm-wide spectral fragments in the range $390-730 \mathrm{~nm}$, or in a chosen part of this range. The width of the spectral fragments depends on the chosen grating and can be changed using the slits of the monochromator. Two regimes were normally used, with 10- and 15-nm-wide slits for the first grating, and with 20- and 30-nm-wide slits for the second.

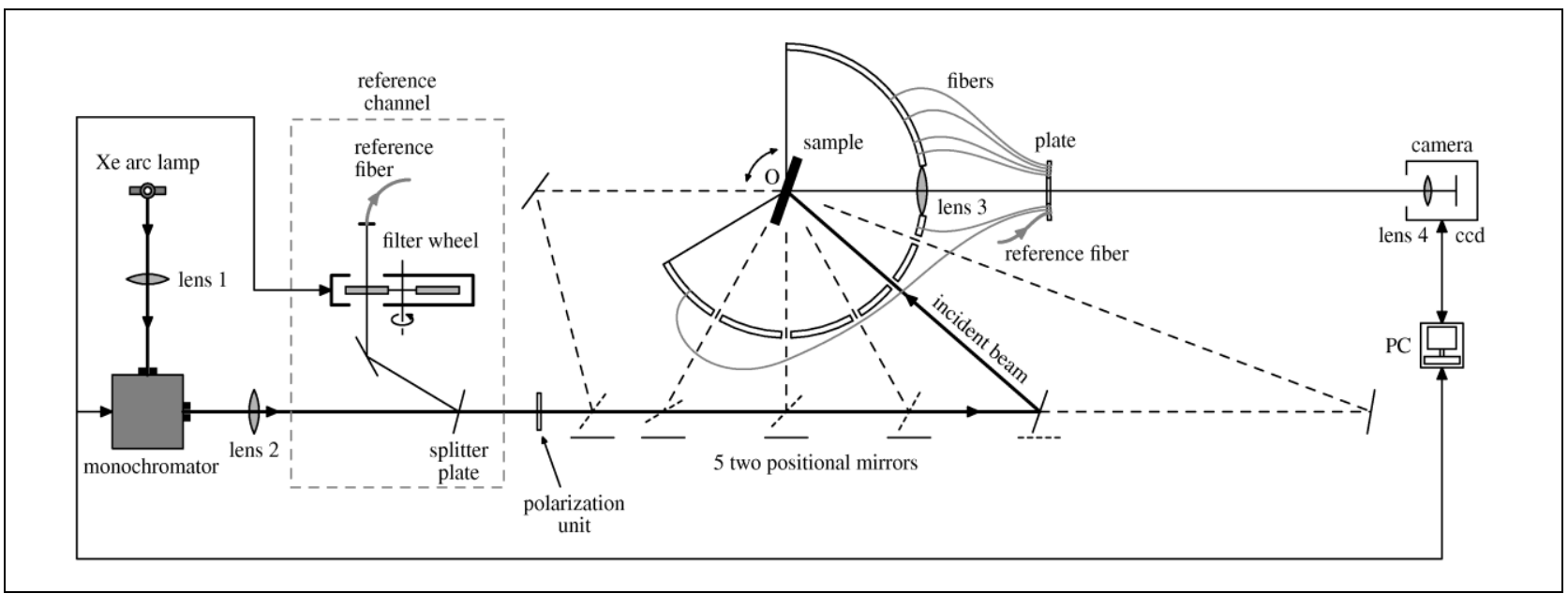

Fig. 1. The schematic of the device

A lens with 150-mm focal length is used as the output collimator (lens2), which focused a 10-times-magnified image of the output slit of the monochromator onto a plane close to the plane of the sample. Behind the lens, a parallel splitter glass plate with one antireflection-coated surface is placed at an angle $\sim 6^{\circ}$ with respect to the beam, which branches $\sim 5 \%$ of the light beam into the reference channel. Further along the reference beam, a computer-directed wheel with a number of certified 
neutral filters is placed which serves to increase the dynamic range of the device. The main part of light passed through the splitter plate get a polarization unit. This is a polarizer of some type to control the polarization of the incident light, or a depolarizer if we needed to imitate naturally polarized light. Then the light passed through the splitter plate and polarization unit is reflected by a mirror system and reaches the sample through one of the inlets situated at the "equator" of the semisphere (see Fig. 1).

The incident beam is situated in the horizontal plane. The plane of the sample is vertical, and the center of the lighted area in this plane coincides with the center $\mathrm{O}$ of the semi-sphere. The sample can be rotated about two axes, the vertical one and the horizontal one, both located in its plane. To change the incident angle, it is sufficient to rotate the sample about the vertical axis that pass through the center $\mathrm{O}$, while using the second degree of freedom of the sample for adjustments. An additional appliance is used to measure the properties of anisotropic samples which allowed rotating the sample about the axis perpendicular to its plane and passing through the center $\mathrm{O}$.

When measuring the BRDF at different incident angles, the beam can pass through one of the 5 inlets on the sphere which allow for incident angles $\sigma=0^{\circ}, 10^{\circ}$, $20^{\circ}, 30^{\circ}, 45^{\circ}$, and $60^{\circ}$. When measuring the BTDF, the beam enters the semi-sphere from the rear side, where there is no wall. So, it is possible to realize a large number of incident angles up to $80^{\circ}$ using a mirror system. Some of these mirrors are twopositional ones and may be turned off if necessary.

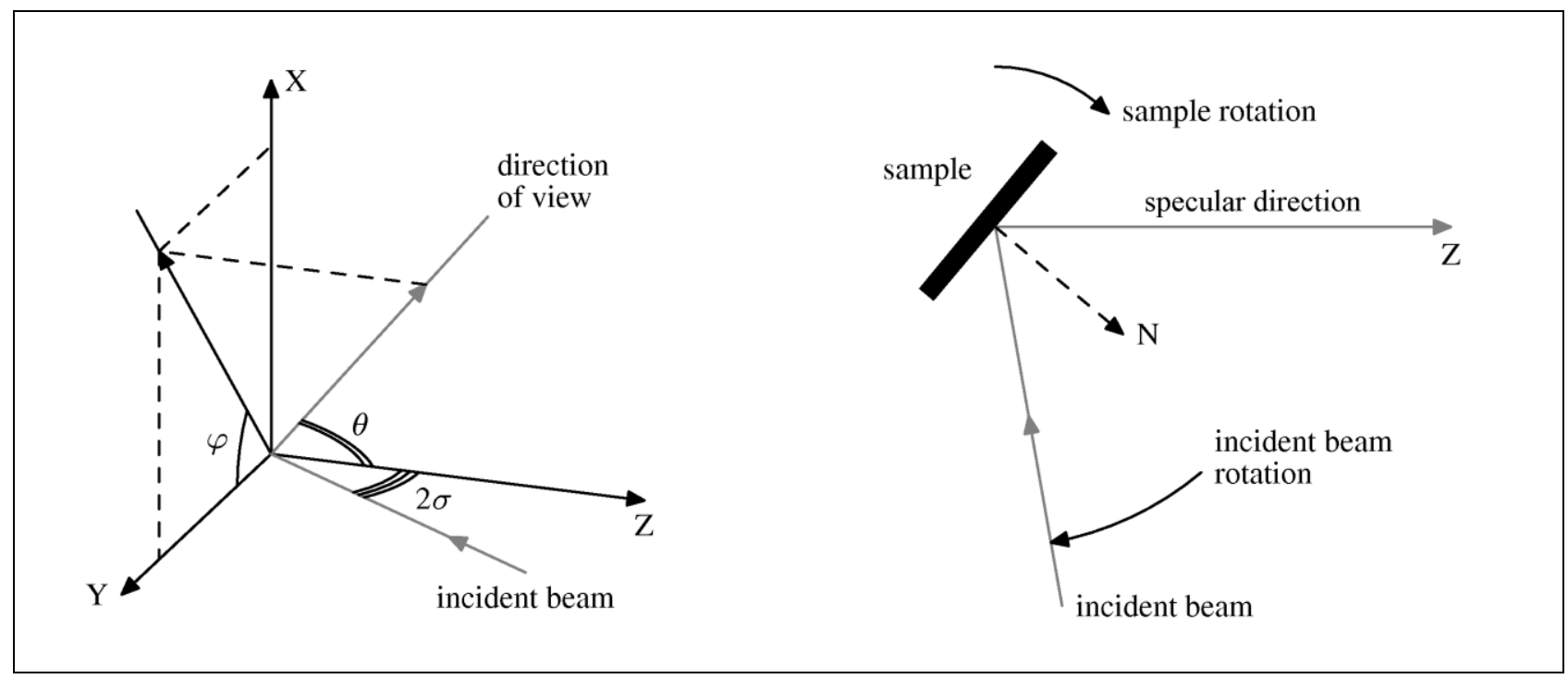

Fig. 2. Coordinate system: left image - directions of incident and reflected light, right image - change in direction of the incident ray of light when the sample is rotating

To describe spatial coordinates of BRDF we use spherical coordinate system conventional for computer graphics applications. It gives the possibility to easily apply the measured functions as input data for such systems. The horizontal axis $\mathrm{Z}$ is the direction of specular reflection. The incident beam and the normal to the sample surface are also situated in horizontal plane. The direction of distributed light (direction of view) is determined by two angles, $\theta \square$ and $\square \varphi$. The angle $\theta$ is formed 
by the direction of view and the axe Z. The angle $\varphi$ is the angle between the incident plane and the plane formed by the axis $\mathrm{Z}$ and the direction of view, fig.2. The angle $\sigma$ between incident beam and the normal to the surface gives the third coordinate. The coordinate system with angles $\sigma, \theta$, and $\varphi$ is used in the computer graphics systems and when modeling intensity of illumination [Ignatenko, 2004, Barladian, 2005].

The practical realization of such coordinate system demands that the $Z$-axis (specular reflection direction) is always directed towards the camera. In our installation, the camera is stationary, and the needed geometry is obtained by simultaneously changing the direction of the incident light and rotating the sample (see Fig. 2b). The Bidirectional Reflectance /Transmittance Distribution of the light reflected within $10^{\circ}$ of the direction of specular reflection is recorded as an image. An 80 -mm-diameter 150 -mm-focal-length collecting lens is used to this effect. It is placed at $200 \mathrm{~mm}$ from the sample in the inlet of the semi-sphere. The angular distribution of the light reflected from the sample in this case is converted into spatial distribution in the focal plane of the lens. The simplest decision then would to project the whole of the reflected light directly onto the CCD matrix of the camera. However, the resulting multiplication of such projection is the product of the angular and the linear ones (since the brightness is conserved). As a result, when using the serial photographic lens, too much space is needed on the CCD matrix, which should also be used to record the reflected light collected by the optical fibers.

To overcome this limitation, a 1-mm-thick 50-mm-diameter glass disc was placed in the focal plane of the lens. The side of disc directed toward the lens is matted. When light falls normally at the disc, the half-width of the indicatrix of reflection is about $15^{\circ}$, and when its incident angle is $10^{\circ}$, the maximum amplitude is reduced by about $15 \%$ while the half-width increases to $18^{\circ}$. At the same time, the maximum of the indicatrix belongs to the continuation of the incident beam. The lens of the CCD camera is placed where the image of the surface of the sample would have been focused in the absence of the matted plate, in the place with maximum light intensity.

The matted surface of the disc is in the focal plane of the lens 3 . Therefore, the angular distribution of the light reflected by the sample is, as previously mentioned, converted into the spatial one. The values of $\theta$ and $\varphi$ are converted into the corresponding coordinates on screen. This means that the light reflected in any point of the lighted area of the sample in the direction given by the angles $\theta$ and $\varphi$ and gathered by the lens 3 will arrive in the same point of the plate which corresponds to these angles (see Fig. 3). This allows us to noticeably increase the angular resolution near the direction of mirror reflection.

Outside the region where the light is gathered by the lens, the scattered light is gathered with the optical fibers. The input ends of the optical fibers are affixed to the inlets drilled into the semi-sphere. Their axes are directed toward the center of the semi-sphere, i.e. towards the center of the lighted region of the sample. Thus, the inlets with the optical fibers are placed so as to create a needed array of the output angles $\varphi$ and $\theta$. 


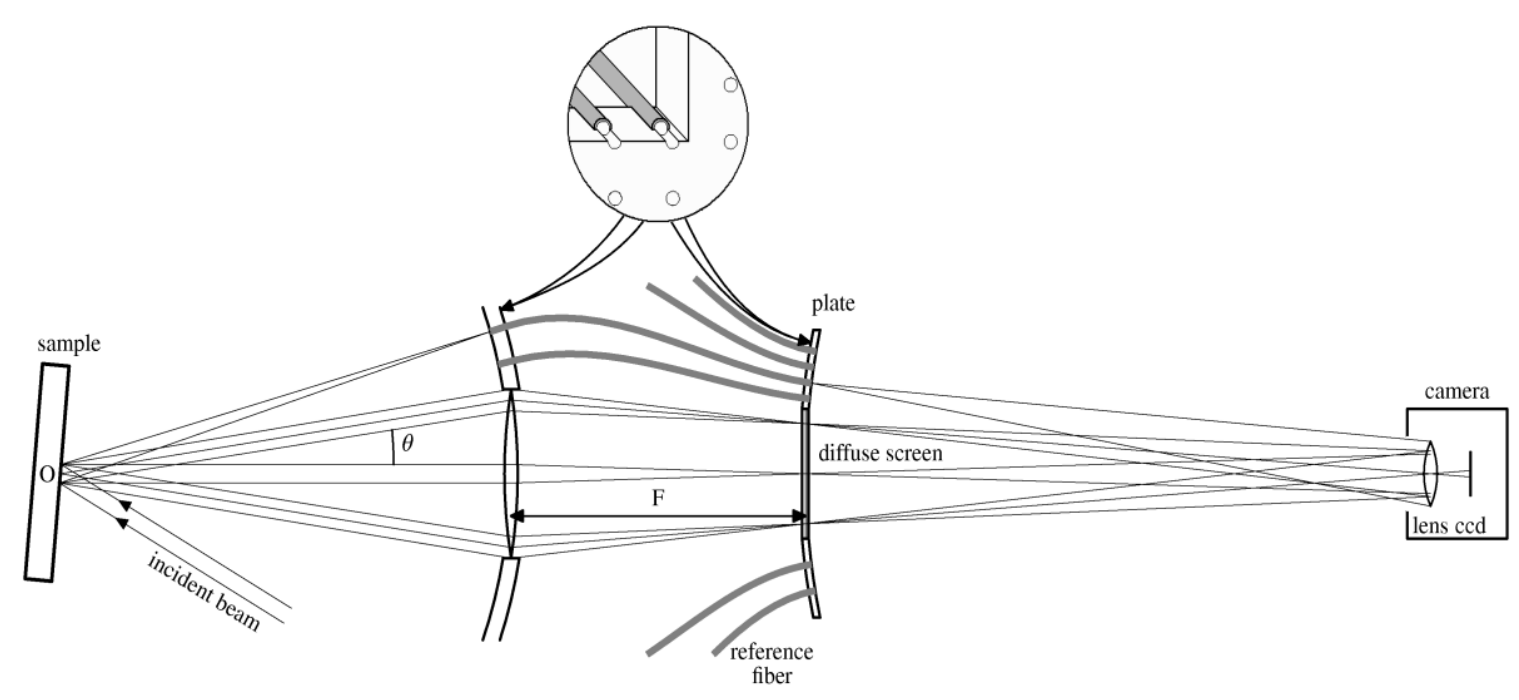

Fig. 3. Scheme of the path of beams from the sample to the CCD camera while converting the angular distribution into spatial

The output ends of the optical fibers that start from the semi-sphere as well as the output end of the optical fiber of the reference channel are affixed to the blackened metal screen. The matted plate described above is placed in the middle of the screen. The side of the screen directed toward the camera has a form of a sphere with a center in the lens of the camera. The Santa Barbara Instruments Group, Inc. ST-5C CCD camera forms the image of the screen, with the plate from matted glass and the output ends of the optical fibers.

We use the matted semi-transparent screen, so BTDF depend only weakly on the incident angle and reflection angles. It allows us to solve two problems at once.

First, the light from the different fragments of the lighted surface of the sample which was reflected at the same angle propagates at different angles after passing through the lens which converts the angular distribution into the spatial one. Yet the matted screen allows equalizing the contributions of these fragments, rendering them proportional to the light intensity on the fragments. Such solution guarantees a relatively uniform gathering of light from the different fragments of the lighted surface by the CCD matrix, even for partial light gathering, which, in turn, lowers the requirements for the homogeneity of lighting of the working surface of the sample by the incident light. This technique is usually applied when using integrating spheres.

Second, the matted plate and the optical fiber have close (similar) BTDFs near the zero incident angle. This lowers the contrast between the brightness of the different sources of reflected light (ones gathered by the lens and the optical fibers). is significant for the light receiver with moderate dynamic range, such as the CCD matrix. As a result, the number of frames necessary for the measurement decreases. The influence of the discharge blooming from over-lighted regions of the CCD matrix decreases too. It concerns the frames whose expositions allow one to provide high-quality measurements with the less bright periphery optical fibers.

The amplitude calibration of the multi-element system is the rather difficult process. The direct approach requires detailed measurements of numerous absolute quantities. There are the spectral brightness of the xenon lamp, the fraction of its light 
reached the monochromator, the transmission of the monochromator and the mirror tract at different wave lengths. There is also the fraction of the light reflected at different angles which is gathered by each optical fiber, passed through it and collected by the camera lens onto the CCD matrix, as well as the same values for the lens path. Therefore, it is reasonable to calibrate the system using a diffusing object with well known light-scattering characteristics. Such approach is preferable in nearly all tasks of measuring the amplitude of the scattered light [Bogomolov \& Letunov], to the difference of light emission measurements.

A sample from MS-20 milk glass is used as a standard, cut in the form of a 60mm-diameter 10-mm-thick disc. This sample was studied in Russia Research Institute of Optical and Physical Measurements and its characteristics certified for normal incident light in the entire visible range $(390-730 \mathrm{~nm})$ at intervals of $10 \mathrm{~nm}$ and at observation angles from $10^{\circ}$ to $85^{\circ}$ at intervals of $5^{\circ}$. Based on these measurements, it is possible to obtain the data for all needed observation angles using a linear approximation. When calibrating the system, the standard is placed perpendicularly to the incident beam, since this is the only position for which its characteristics had been measured and certified. With the etalon in this position, a complete measurement cycle is done on every wave length. The results are recorded and used to normalize all the other data. Note that to simplify the measurements and to increase their accuracy, it might be preferable to use a standard whose characteristics were previously recorded for every incident angle, which would allow us to place the standard the same way as the sample. However, the known producers only provide their diffuse standards with the spectral dependences of the total reflection integral for the incident angle of either $0^{\circ}$ or $8^{\circ}$, which is known to be insufficient in our conditions.

The image formed by the CCD camera is then recorded by the computer which provides the calculations for the raw experimental data and their post-processing. One of the functions of the computer is to control the devices constructing the complex: the monochromator, the wheel with filters, and the camera.

\section{Measurement process.}

The device records simultaneously all the components of the BDF allowed by its construction for a given wave length at a given incident angle of the light beam. For a fixed incident angle, the entirety of the spectral range is scanned using the computer-operated monochromator. When doing this, frames are recorded for every wave length with a number of expositions whose values are determined by the operating program depending on the brightness of the sample. These actions are the basis of the measuring cycle for a given incident angle and they are performed automatically. The changes of the incident angle (by changing the positions of the mirrors) are done manually. The change of the incident angle for BTDF is achieved by rotating the sample, and for BRDF, by simultaneously rotating the sample and changing the trajectory of the incident beam (see Fig. 2).

When measuring the BRDF for a typical isotropic sample in full-color mode (35 spectral points), the time needed for manual changes is about an hour. After 
optimizing the device scheme, the time needed for such changes decreased to about 20 minutes.

A relatively easy and illustrative way to demonstrate the merits of our device is to present measurement results for samples with a well-defined spike of back scattering.

Figure 4 presents the typical measurement results for such a sample in the spike region for incident angle $\sigma=20^{\circ}$ and wavelength $650 \mathrm{~nm}$ before postprocessing. Indicatricies are presented for reflection near $\theta=40^{\circ}$ with azimuthally angles $\varphi=0^{\circ}, 12.25^{\circ}$, and $25.5^{\circ}$.

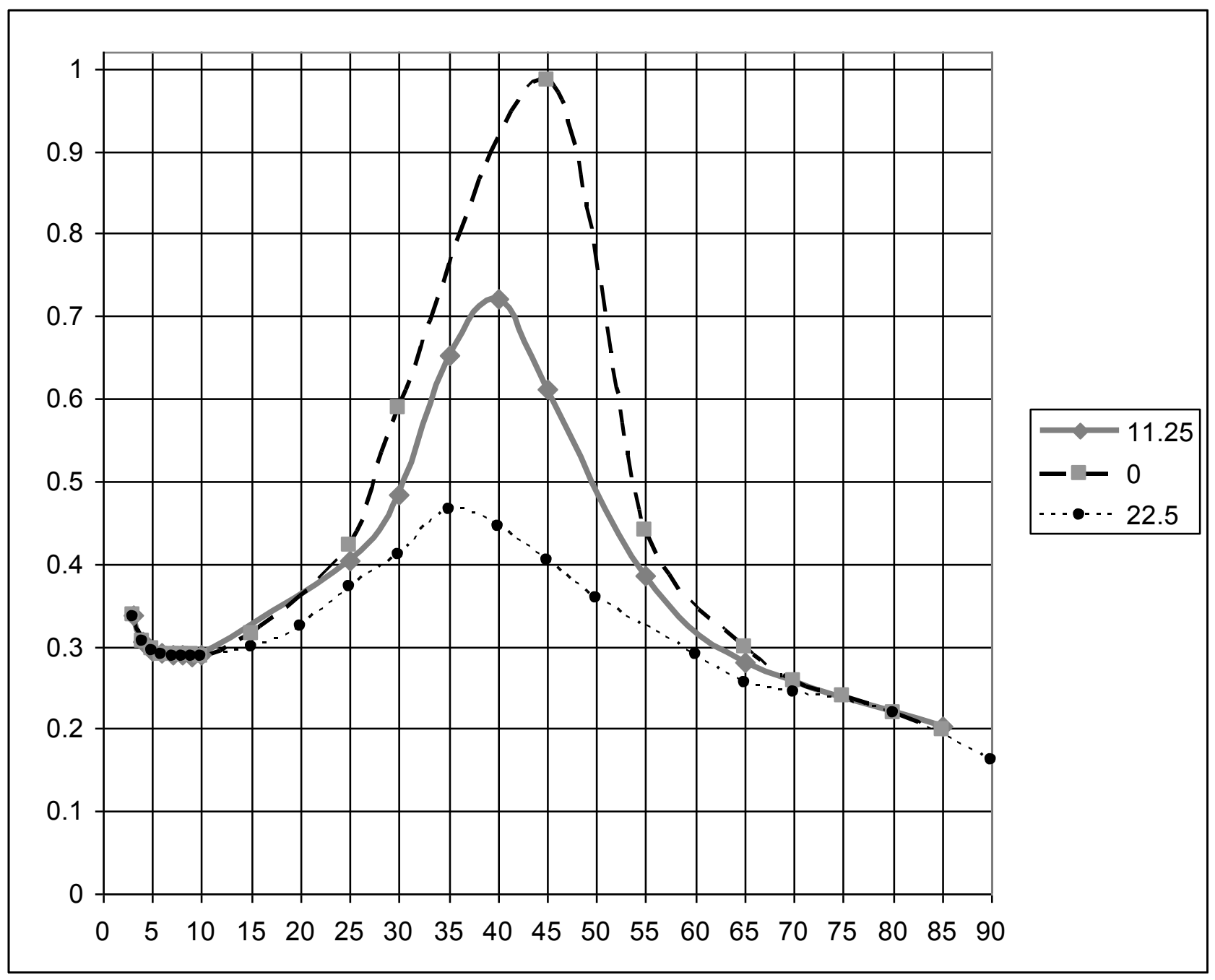

Fig. 4. Measurement results for samples with scattering

The presented data are some part of the more then 5000 lines of the output file of full-color measurement. The errors calculated from the internal control of reciprocity at $\varphi=0^{\circ}$ are $3-5 \%$, which confirms the high quality of our measurements.

At this time, the device is measuring the data from typical samples in normal operation regime. The time of full-color measurement of the properties of an isotropic sample (without post-processing) was decreased to 3-4 hours. 
Anisotropic and especially polarizing samples from complex materials need a high number of local measurements; the post-processing of the obtained data is also more complicated. The too-dark samples demand longer exposures. Samples in which the maximum of the reflected light beam is near the direction of mirror reflection also demand more time to measure their BDF, since they cause blooming, an effect of creating a halo in the CCD cameras. In this case, it is necessary to measure the lens region separately, then cover the lens by an opaque screen and take the measurements from optical fibers. In some cases, it is possible to obtain reliable data from inhomogeneous surfaces, as well as the front surfaces of semi-transparent samples, by excluding the noise introduced by their rear surfaces. It is obvious that all these cases demand more measurement time.

\section{Technical features.}

The spectral range of our device is $390-730 \mathrm{~nm}$. The dynamic range of BDF is $0.05-2500$. The reproducibility of the results is $1-3 \%$. The accuracy of the results is $4-8 \%$. The mean angular resolution is $3^{\circ}$, it is $0.5^{\circ}-1.5^{\circ}$ in the range up to $10^{\circ}$ from the direction of mirror reflection when measuring BRDF, and $1.5^{\circ}-8^{\circ}$ in other directions. The size of the light spot is $0.7-2 \mathrm{~cm}$ (depending on incident angle). The minimal sample size is $5 \mathrm{~cm}(\mathrm{~L}) \times 3 \mathrm{~cm}(\mathrm{H})$, and the maximum size is 20 $\mathrm{cm}(\mathrm{L}) \times 15 \mathrm{~cm}(\mathrm{H}) \times 1 \mathrm{~cm}(\mathrm{Th})$ (where $\mathrm{L}$ is its length, $\mathrm{H}$ is its height and $\mathrm{Th}$ is its thickness).

The construction of the measurement complex is intended for flat samples. The curvature of the surface negatively affects the accuracy of the results, yet curvature radii of $300 \mathrm{~cm}$ and more are acceptable, since they do not noticeably increase the existing measurement error.

\section{Discussion of results. Future plans.}

During the period of test and normal operation, the device proved an efficiency of general approach and its technical realization. Nevertheless, we see several soft spots. The fourth spatial valuable for anisotropic surfaces may be accounted only if some type of symmetry exists and is known in advance. In the area of fibers, the results of measurements are discrete with big step. It means that the result of postprocessing depends on the methods of interpolation. Then, for calibration, we have only the data for normal incident beam; etalons for other directions could provide the higher accuracy, but they are not available. Finally, the manual part of work is still too large.

Some of these limitations may be removed in the next version. In order to improve the parameters of the device, we plan on including a second CCD camera. This will enable us to separate the measurements from the fiber and lens regions, since the measurement conditions are too different for these two cases. It will be possible to realize an autonomous light gathering system with higher power for each of these regions. It makes it possible to optimize the choice of exposures to the separate recording of the light intensities of different regions. As a result, it allows 
increasing the accuracy and decreasing the time of the measurements. It also allows using additional fibers. With the decrease of the instrumental time, it will be necessary to increase the level of automatization of our measurements without rendering them less flexible. A step in this direction is the automatization of the change of direction of the incident beam and the orientation of the sample. The latter should both accelerate the measurement process and decrease the angular error of the sample orientation 2-3 times and become a useful and exact mean of adjustment of the device. The improved possibilities of tuning the optical system should, in turn, facilitate a fuller realization of the above mentioned advantages of such modernization.

\section{Conclusions.}

A new concept of the optical scheme of the measurements was developed which allows us to simultaneously record the signals from the light fibers and the image formed by the lens in such a way that the angles become spatial coordinates. The use of the CCD camera give the possibility to simultaneously record light from a great number of points, which renders the measurement time practically independent of the number of light fibers used, i.e. of the number of the spatial components of BDF. While the dynamic range of the measured intensities is more than five orders of magnitude, the achieved measurement accuracy is $4-8 \%$, and is acceptable for most tasks.

The obtained measurement results were used in the system for real-time synthesis of realistic images [Ignatenko et al, 2004] and in the Internet service for modeling the lighting and synthesis of realistic images [Barladian et al, 2005]. They can also be used in similar systems. The used of the measurement data in construction systems and modeling of lighting optical devices based on the Monte Carlo calculation of the light propagation [Voloboy et al, 2005] allowed us to obtain results with error about $5-10 \%$ as compared to the measured characteristics of real devices.

\section{Acknowledgements}

This work was supported by the Russian Leading Scientific Schools Foundation, project NSh- 8129.2010.9, RFBR, grants 09-01-00299, 10-01-00302 and 11-0100870, and by INTEGRA Inc.

\section{References}

B. K.P. Horn, "Robot Vision", MIT Press and McGraw-Hill, Cambridge, MA, 1986 Ward G.J., Measuring and Modeling Anisotropic Reflection. Computer Graphics, Volume 26, Number 2, July 1992

Letunov A.A, Barladian B.H., Zueva E.Yu., Veshnevetc V., Soldatov S. CCD-based device for BRDF measurements in computer graphics. The 9th International Conference on Computer Graphics \& Vision. Russia, Moscow, 1999, pp.129135 
A.A.Letunov, V.A.Galaktionov, B.H.Barladian, E.Yu.Zueva, V.P.Veshnevetc, S.A.Soldatov Light scattering measurement complex based on video camera for use in computer graphics Preprint, Inst. Appl. Math., the Russian Academy of Science. № 71, 2000 (rus)

Letunov A.A., Barladian B., Galaktionov V. A.,Ershov S. V. Voloboy.A., Zueva E. The device for measurements of spectral - dimensional distribution of light, dispersing by surfaces. $/ 22^{\text {nd }}$ DAAAM World Symposium 2011 Intelligent Manufactoring \& Automation Vienna, Austria - November 2011. /DAAAM International Scientific Book 2011 ISNB 978-3-901509-84-1, ISSN 1726 9687/EBSCO

Karner K.F., Mayer H., Gervautz M., An Image based Measurement System for Anisotropic Reflection. Eurographics'96, Computer Graphics Forum, 15(3), pp. $119-128$

Foo S.C., A Gonioreflectometer for Measuring Bidirectional Reflectance of Material for Use in Illumination Computation. Cornell University, Thesis, 1997

W. Matusik, H. Pfister, M. Brand, L. McMillan. Efficient Isotropic BRDF Measurement. Eurographics Symposium on Rendering, 2003. Mitsubishi Electric Reasearch Laboratories, TR2003-80

Yasuhiro Mukaigava, Kohei Sumino, Yasushi Yagi. Multiplexed Illumination for Measurng BRDF Using Ellipsoidal Mirror and a Projector. Y.Yagi et al (eds.). ACCV 2007, Part II., LNCS 4844. pp. 246-257, 2007

Bogomolov G.D. \& Letunov A.A. Amplitude calibration of systems for laser plasma diagnostics by rotational Raman scattering in hydrogen and deuterium. Sov.J. Plasma Physics, № 5(6), 1979, p. 774-776

Ignatenko A., Barladian B., Dmitriev K., Ershov S., Galaktionov V, Valiev I., Voloboy A., A Real-Time 3D Rendering System with BRDF Materials and Natural Lighting. The 14-th International Conference on Computer Graphics and Vision GraphiCon-2004, Moscow, September 6-11, 2004. Conference Proceedings, pp. 159-162

Barladian B.H., Voloboy A.G., Vyukova N.I., Galactionov V.A, Deriabin N.B. The lighting simulation and photorealistic image synthesis using Internet technologies. "Programming", № 5, 2005

Voloboy A.G., Galactionov V.A., Ershov S.V., Letunov A.A., Potemin I.S. The device-software complex for light-scattering behavior of surface measurements. Information technologies and computational systems, 2006. № 4, p24-39 (rus)

Voloboy A.G., Galactionov V.A., Dmitriev K.A., Kopylov E.A.. Bi-directed ray tracing for lighting integration by the quasi Monto-Carlo methods. "Programming", № 5, 2005 p. 25-34 (rus)

Voloboy A.G., V'ukova N.I., Galaktionov V.A., Ershov S.V., Letunov A.A, Potemin I.S. An Installation for Measurement of Light Scattering Properties of Surfaces Keldysh Institute of Applied Mathematics RAS, preprint № 108, 2005 (rus) 